\title{
Migraine: treatments, comorbidities, and quality of life, in the USA
}

This article was published in the following Dove Press journal:

Journal of Pain Research

12 August 2015

Number of times this article has been viewed

\author{
Christopher D Malone' \\ Amrita Bhowmick ${ }^{2}$ \\ Amy B Wachholtz' \\ 'Department of Psychiatry, University \\ of Massachusetts Medical School, \\ Worcester, MA, ${ }^{2}$ Health Union, \\ Philadelphia, PA, USA
}

\begin{abstract}
This study sought to characterize the experience of stress, treatment patterns, and medical and disability profile in the migraineur population to better understand how the experience of migraines impacts the social and psychological functioning of this group. A 30-minute self-report survey was presented via a migraine-specific website with data collection occurring between May 15 and June 15, 2012. Recruitment for the study was done through online advertisements. In total, 2,907 individuals began the survey and 2,735 met the inclusion criteria for the study. The sample was predominantly female (92.8\%). Migraine-associated stress was correlated with length of time since first onset of symptoms $(P<0.01)$ and number of symptoms per month $(P<0.01)$. Disorders related to stress, such as depression $(P<0.01)$ and anxiety $(P<0.01)$, were also positively correlated with the measured stress resulting from migraines. Migraine-associated stress must be understood as a multidimensional experience with broader impacts of stress on an individual correlating much more highly with negative mental and physical health profiles. Stress resulting from frequent migraine headaches may contribute to the development of medical and psychological comorbidities and may be a part of a cyclical relationship wherein stress is both a cause and effect of the social and medical impairments brought about by migraine.
\end{abstract}

Keywords: characteristics, migraine, headache, stress, treatment satisfaction

\section{Introduction}

Migraine headache is a chronic pain condition that afflicts millions of Americans, with prevalence estimates ranging between $16.2 \%$ and $22.7 \%$ for USA adults aged 18 years or older. ${ }^{1}$ Migraines are disabling, with a majority of migraineurs reporting impairments in their ability to engage in work activities, perform household chores, attend social activities, and maintain relationships due to their migraines. ${ }^{2}$ The social costs of migraine are felt in the form of lost productivity as well as increased health care costs. ${ }^{3}$ The disorder is further complicated by the multiple and varied symptoms that have been reported, including sweating, allodynia, photophobia, phonophobia, vomiting, and nausea. ${ }^{4}$

In light of the high prevalence rate and significant impairment of daily living, it is unsurprising that patients use many treatment approaches to alleviate the impact of migraine. Pharmacological treatments are common, with both abortive and prophylactic medications used; however, these medications are burdened by side effects and cost. ${ }^{5}$ Non-pharmacological interventions, such as behavioral therapy, ${ }^{6}$ relaxation therapy, and cognitive behavioral therapy, have also been employed, with varying degrees of success. ${ }^{5}$ Despite the variety of treatment approaches available, migraineurs report that they feel their treatment is insufficient or unsatisfactory. ${ }^{7}$
Correspondence: Amy BWachholtz Department of Psychiatry, University of Massachusetts Medical School, 55 Lake Avenue North, Worcester, MA 01655 , USA

Tel + I 5083342164

Email amy.wachholtz@umassmemorial.org 
Frequent migraine headaches also impose a heavy psychological burden on migraineurs. Both stress and anxiety levels are higher among migraineurs when compared with healthy controls. ${ }^{8}$ Other research has found a reinforcing cyclical relationship between stress and migraine headaches. ${ }^{9}$ The relationship between stress and migraine may also have a deeply personal component, as suggested in recent findings, ie, an individual's response to stressors, rather than the stressors themselves, may be the predisposing factor to developing migraine. ${ }^{10}$ Previous research has treated the experience of stress in the context of migraine headache as principally a prodromal indicator of onset of migraine and a strictly internal experience on the part of the migraineur. ${ }^{8}$

The experience of migraines is further complicated by comorbid medical and psychological disorders. Psychological disorders, especially depression and anxiety, have been found to be prevalent among migraineurs. ${ }^{2}$ Chronic pain, stroke, gastrointestinal disorders, cardiovascular disease, and other medical conditions have also been found to be more prevalent in migraineurs compared with the general population. ${ }^{11}$ Given the high prevalence of migraine headaches, it is unsurprising that a large variety of comorbid medical and psychological disorders is found in the migraine population.

The high social and personal costs of migraine as well as complications in delivering effective migraine treatment emphasize the need to understand treatment patterns in this highly prevalent clinical population. The current study extends the available literature by describing the relationship between stress, treatment patterns and satisfaction, and comorbid disorders in a large online sample of migraineurs. This study elaborates on previous research into migraineassociated stress by viewing the stress experience as a social as well as internal impact of migraine headaches.

\section{Patients and methods}

\section{Recruitment}

Participants were recruited from a well-known online migraine headache resource. Adults aged 18 years or older and currently living in the USA were invited to participate. The presence of migraines was validated through agreement with the statement "Have you ever been diagnosed with migraine headaches by a physician?"

\section{Procedure}

This study was approved by the University of Massachusetts institutional review board. Between May 15 and June 15 2012 , an online survey was presented via a migraine-specific community website. ${ }^{12}$ Participants were informed that their participation was voluntary, information would be collected anonymously, the anticipated completion time of the survey, and that they would not receive compensation for participating. No personal identifiers were collected nor solicited from participants. The survey contained questions related to demographic information, migraine history and symptoms, social information, and treatment history and satisfaction. The survey host used embedded cookies to prevent any participant from taking the survey multiple times. The survey employed an adaptive methodology. Data were collected by the survey host and stored on a secure server.

A composite variable termed "negative live events" was created using items from the survey that examined specific areas of life impacted by participant migraineurs. The negative life events score was further divided into variables examining internal areas of life, such as mental state and well-being, and external areas of life, such as social relationships.

\section{Data analysis}

All data analysis was performed using Statistical Package for the Social Sciences version 22.0 (IBM Corporation, Armonk, NY, USA). Participant demographics were analyzed using descriptive statistics and analysis of variance, and Pearson's $R$ correlation analyses were used to examine relationships among the survey data. Path analyses were conducted to examine differential relationships among the length of time since onset of migraine symptoms, the number of reported comorbidities, the frequency of migraine symptoms, the number of migraine symptoms, and composite scores reflecting impacts resulting from migraines (negative life impact internal score and negative life impact external score).

\section{Results \\ Participants}

The survey was started by 2,907 individuals. A total of 172 individuals were excluded due to: being younger than 18 years $(n=32)$, not diagnosed with migraines $(n=19)$, or not currently living in the USA ( $n=121)$. Of the remaining 2,735 individuals who started the survey, 2,632 completed it (giving a completion rate of $96.2 \%$ ). The mean time taken to complete the survey was 23 minutes ( \pm standard deviation 1 minute 25 seconds). Due to the use of adaptive survey methodology, participants did not answer questions that were not pertinent to them, resulting in a small variation in the number of responses per question. 


\section{Demographics}

The sample was $92.8 \%$ female and nearly half of the sample (47.8\%) was aged $40-54$ years, which is demographically similar to national rates for migraineurs. The majority of the sample $(75.9 \%)$ had experienced their migraine symptoms for more than 10 years. The majority of the sample (68.3\%) was diagnosed with chronic migraine. A quarter of the sample (25.1\%) reported experiencing migraine symptoms on more than 20 days per month; the remainder of the sample experienced migraine symptoms less frequently. Additional demographic information can be found in Table 1.

\section{Comorbid disorders}

Table 2 shows the data relating to reported comorbid disorders. Depression and anxiety were found to be the most commonly reported comorbid conditions, at $63.8 \%$ and $60.4 \%$, respectively. Chronic (non-headache) pain was reported by $39.5 \%$ of the sample. All remaining disorders were reported by less than onethird of the sample. A minority of participants (16.4\%) reported suffering from a disorder not listed, and of those participants, the vast majority reported multiple comorbid disorders (89.9\%).

Table I Patient demographics and disease characteristics

\begin{tabular}{|c|c|}
\hline & Patient, n (\%) \\
\hline \multicolumn{2}{|l|}{ Sex } \\
\hline Male & $196(7.2)$ \\
\hline Female & $2,514(92.8)$ \\
\hline \multicolumn{2}{|l|}{ Age, years } \\
\hline$<18$ & $18($ Excl)* \\
\hline $18-24$ & $190(6.9)$ \\
\hline $25-39$ & $921(33.7)$ \\
\hline $40-54$ & I,307 (47.8) \\
\hline$>55$ & $317(11.6)$ \\
\hline \multicolumn{2}{|c|}{ Length of time since first migraine symptoms } \\
\hline$<$ I year & $38(1.4)$ \\
\hline I-5 years & $267(9.9)$ \\
\hline $6-10$ years & $349(12.9)$ \\
\hline $10+$ years & $2,056(75.9)$ \\
\hline \multicolumn{2}{|c|}{ USA resident } \\
\hline Yes & $2,735(95.6)$ \\
\hline No & $|2|($ Excl)* \\
\hline \multicolumn{2}{|c|}{ Days per month migraine symptoms $\geq 4$ hours ( $n$ ) } \\
\hline $\mathrm{I}-4$ & $464(17.1)$ \\
\hline $5-9$ & $564(20.8)$ \\
\hline $10-14$ & $513(18.9)$ \\
\hline $15-19$ & $488(18.0)$ \\
\hline $20+$ & $681(25.1)$ \\
\hline \multicolumn{2}{|c|}{ Ever diagnosed with migraine by physician } \\
\hline Yes & $2,503(98.2)$ \\
\hline No & $19($ Excl)* \\
\hline \multicolumn{2}{|c|}{ Ever been diagnosed with chronic migraine } \\
\hline Yes & $1,709(68.3)$ \\
\hline
\end{tabular}

Note: *These patients were not included in the percentage basis for the final analyses. Abbreviation: Excl, excluded.
Table 2 Comorbid disorders

\begin{tabular}{ll}
\hline Comorbid disorder & Patient, $\mathbf{n}(\%)$ \\
\hline Depression & $1,4 I I(63.8)$ \\
Anxiety & $1,328(60.4)$ \\
Stroke & $55(2.5)$ \\
Epilepsy & $58(2.6)$ \\
Hypertension & $390(17.6)$ \\
Diabetes & $124(5.6)$ \\
Bipolar & $145(6.6)$ \\
IBS & $647(29.3)$ \\
Chronic pain & $874(39.5)$ \\
Fibromyalgia & $360(16.3)$ \\
Thyroid disease & $357(16.1)$ \\
Chronic fatigue & $574(26.0)$ \\
Asthma & $44 I(19.9)$ \\
COPD & $51(2.3)$ \\
Autoimmune disorder & $116(5.2)$ \\
Any comorbid disorder reported & $476(16.4)$ \\
Reported comorbidities & \\
I & $338(71.0)$ \\
2 & $90(18.9)$ \\
3 & $28(5.9)$ \\
4 & $12(2.5)$ \\
5 & $5(1.1)$ \\
$6-I I$ & $3(0.6)$ \\
\hline
\end{tabular}

Abbreviations: COPD, chronic obstructive pulmonary disease; IBS, irritable bowel syndrome.

\section{Negative life events}

Nearly three quarters $(74.1 \%)$ of the sample reported that people in their social network do not believe that the migraines are as severe as the participants report. The migraineurs reported that their employer knew about their migraines $(54.8 \%)$ and the migraines negatively impacted their professional advancement (50.2\%).

A composite variable was created by summing the number of negative life events that participants endorsed in order to examine the extent to which migraine headaches impact their life (Cronbach's $\alpha=0.848$ ). The negative life event score was found to have a mean of 4.023 (standard deviation 27; median 4; range $0-13$ ). The results of questions identifying the impact of migraine on personal aspects of life can be found in Table 3.

The negative events were also examined according to whether the migraineurs perceived that the negative impact was caused by internal (psychoemotional) stress factors or external (social-interactive) stress factors. The negative life impact internal score was composed of questions asking about the migraineurs' feelings of worry or shame about their migraine headaches. The negative life impact external score was composed of questions examining the social, professional, and relational impact of migraines. Pearson's $R$ correlational coefficient path analyses examining the relationship between the characteristics of migraine and the internal 
Table 3 Negative life events

\begin{tabular}{ll}
\hline Event & Patient, n (\%) \\
\hline People don't believe my migraines are severe & $\mathrm{I}, 782(74 . \mathrm{I})$ \\
I am constantly worried about disappointing people & $\mathrm{I}, 38 \mathrm{I}(57.4)$ \\
Employer knows that I suffer from migraines & $\mathrm{I}, 474(54.8)$ \\
Impacted my professional advancement & $\mathrm{I}, 207(50.2)$ \\
Migraines affect my ability to maintain relationships & $\mathrm{I}, 027(42.7)$ \\
Migraines impact my relationship with my & $\mathrm{I}, 029(42.8)$ \\
child/children & \\
I feel like others blame me for my migraines & $966(40.2)$ \\
Sometimes I blame myself for my migraines & $874(36.4)$ \\
Friends/family/colleagues treat me differently because & $807(33.6)$ \\
of my migraines & \\
Doctors treat me differently because of my migraines & $747(3 \mathrm{I} .1)$ \\
Embarrassed & $613(25.5)$ \\
I have lost a job due to migraines & 6 I $2(25.5)$ \\
I have lost friends due to my migraines & $476(19.8)$ \\
Migraines contributed to my divorce/separation & I83 (7.6) \\
\hline
\end{tabular}

and external impacts of migraine can be found in Figures 1 and 2. Positive correlations were found between all examined variables and both the negative life impact internal score and negative life impact external score.

\section{Migraine frequency and symptoms}

Migraine frequency data can be found in Table 4 . The most commonly reported symptoms of migraine experienced by the sample were head pain (98.0\%) and sensitivity to light (91.1\%). Participants who reported a higher frequency of migraine symptoms were also more likely to report more negative life impact due to migraine. Seventy-five percent of participants who were found to have a negative life event score of 10 or higher also reported 15 or more migraine symptoms per month.

\section{Migraine triggers}

Most of the participants (78.2\%) were able to identify at least one trigger for their migraine headaches. Of those participants who did identify a trigger, $78.3 \%$ identified environmental causes (such as weather) and $76.7 \%$ identified stress as a trigger. Of those participants who identified triggers for their migraines, $88.3 \%$ reported that they take steps to avoid those triggers. Additional results are reported in Table 5.

\section{Migraine treatments}

In total, $96.7 \%$ of the sample reported consulting a physician specifically for treatment of migraine headaches at some point and $81.3 \%$ of the sample were currently seeing a physician about their migraine (Figures 3, 4 and Table 6). Of those who do not see a physician about migraine, $47.7 \%$ reported financial cost or health insurance to be the preventing factor. Approximately half of the sample (45.5\%) reported that they avoided taking a prescribed migraine medication due to the cost. Ninety percent of the sample reported trying a prescription oral migraine medication at some point and $67.2 \%$ reported currently being treated with a prescription oral migraine medication. Approximately three quarters of the sample (73.3\%) reported withholding medication to save for later. Participants also reported behavioral therapies

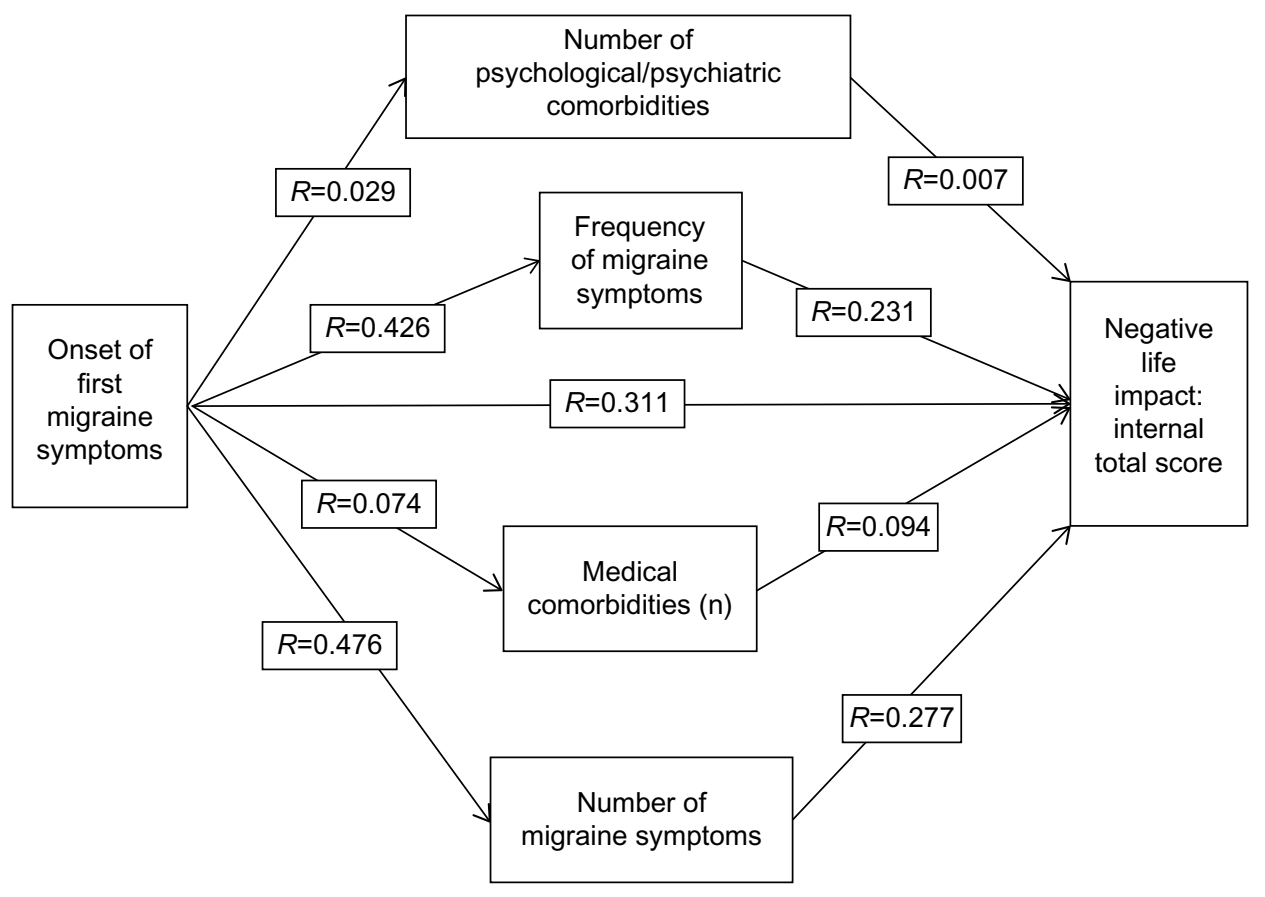

Figure I Path analysis of negative internal life impacts. 


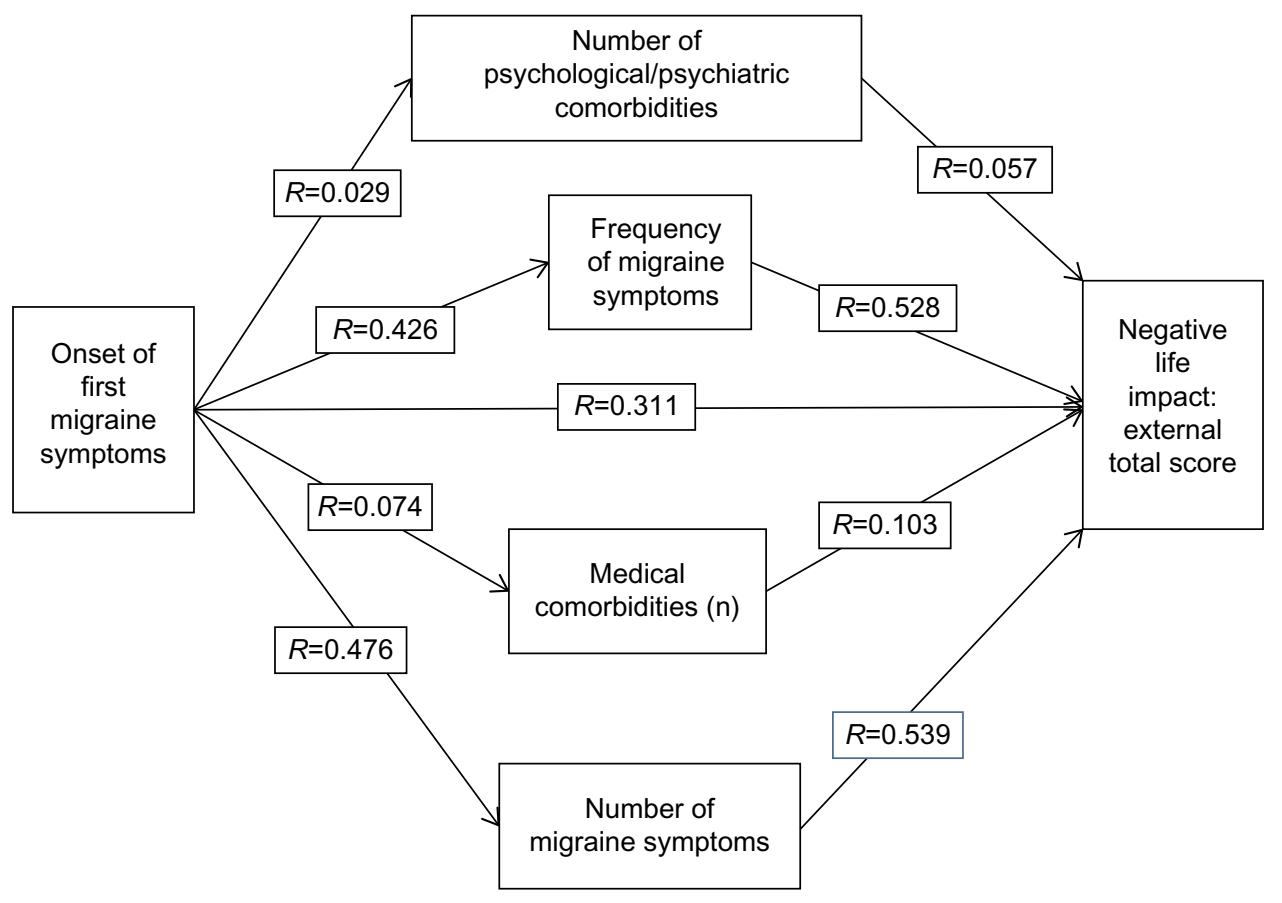

Figure 2 Path analysis of negative external life impacts.

that they had tried, with "dark room" (92.2\%) and "hot/cold therapy" $(75.2 \%)$ being the most commonly tried; however, many participants report initiating a behavioral or complementary therapy (eg, yoga), but not continuing with the practice in the long term.

\section{Treatment satisfaction}

When asked about their current migraine treatment, most individuals appeared to be less than satisfied with their treatment. Approximately one-third of participants (31.2\%) reported feeling "neutral" on satisfaction and a further

Table 4 Migraine symptom frequency

\begin{tabular}{|c|c|c|c|c|c|c|}
\hline & \multicolumn{6}{|c|}{ Days per month impacted by headache symptoms for $\geq 4$ hours, patients, $n(\%)$} \\
\hline & I-4 & 5-9 & $10-14$ & $15-19$ & $20+$ & Total \\
\hline \multicolumn{7}{|l|}{ Sex } \\
\hline Male & $50(25.5)$ & 37 (18.9) & $36(18.4)$ & $22(11.2)$ & $5 I(26.0)$ & $196(7.2)$ \\
\hline Female & $414(16.5)$ & $527(21.0)$ & $477(19.0)$ & $466(18.5)$ & $630(25.1)$ & $2,5 \mid 4(82.8)$ \\
\hline \multicolumn{7}{|l|}{ Symptom } \\
\hline Head pain & $419(16.4)$ & $532(20.8)$ & 487 (19.1) & 47I (18.5) & $643(25.2)$ & $2,552(98.0)$ \\
\hline Sensitivity to light & $400(16.8)$ & $476(20.1)$ & 455 (19.2) & $440(18.5)$ & $603(25.4)$ & 2,374 (9I.I) \\
\hline Sensitivity to sound & $339(15.6)$ & 431 (19.8) & 421 (19.4) & $410(18.9)$ & $57 \mid(26.3)$ & $2,172(83.4)$ \\
\hline Difficulty concentrating & $299(14.3)$ & $416(19.9)$ & 409 (19.6) & $392(18.8)$ & $574(27.5)$ & $2,090(80.2)$ \\
\hline Nausea/vomiting & $334(16.3)$ & $4 I 5(20.3)$ & $388(19.0)$ & $393(19.2)$ & $517(25.3)$ & $2,047(78.6)$ \\
\hline Fatigue & $253(13.0)$ & 401 (20.7) & $378(19.5)$ & $376(19.4)$ & $533(27.5)$ & I,94I (74.5) \\
\hline Neck pain & $262(14.0)$ & $380(20.2)$ & $346(18.4)$ & $378(20.1)$ & $5 I I(27.2)$ & I,877 (72.I) \\
\hline Sensitivity to smell & $224(13.6)$ & $312(18.9)$ & $306(18.5)$ & $343(20.8)$ & 465 (28.2) & $1,650(63.3)$ \\
\hline Mood changes & 197 (12.3) & $313(19.6)$ & $323(20.2)$ & $330(20.6)$ & $438(27.4)$ & $\mathrm{I}, 60 \mathrm{I}(6 \mathrm{I} .5)$ \\
\hline Dizziness/lightheadedness & $216(13.6)$ & $312(19.6)$ & $297(18.7)$ & $313(19.7)$ & $450(28.3)$ & $\mathrm{I}, 588(6 \mathrm{I} .0)$ \\
\hline Migraine aura & $286(19.0)$ & $303(20.1)$ & $285(18.9)$ & $280(18.6)$ & $352(23.4)$ & $\mathrm{I}, 506(57.6)$ \\
\hline Visual changes & $233(16.8)$ & $251(18.0)$ & $262(18.8)$ & $278(20.0)$ & $367(26.4)$ & I,39I (53.4) \\
\hline Weakness & $169(14.2)$ & $219(18.4)$ & $222(18.7)$ & 235 (19.7) & $345(29.0)$ & I, I90 (45.7) \\
\hline Numbing/tingling & $133(14.6)$ & $133(14.6)$ & $164(18.1)$ & $183(20.2)$ & $295(32.5)$ & 908 (34.9) \\
\hline Diarrhea/constipation & $86(10.9)$ & $134(17.0)$ & |4| (17.9) & 178 (22.6) & 247 (31.4) & $786(30.2)$ \\
\hline Vertigo & $87(11.4)$ & $125(16.4)$ & I 52 (20.0) & $150(19.7)$ & $247(32.5)$ & $76 I$ (29.2) \\
\hline Puffy eyelid & $58(9.5)$ & $122(20.1)$ & $107(17.6)$ & 135 (22.2) & $186(30.6)$ & $608(23.2)$ \\
\hline Food cravings & $44(8.1)$ & $99(18.1)$ & $116(21.4)$ & $123(22.7)$ & I6I (29.7) & $542(20.8)$ \\
\hline \multicolumn{7}{|l|}{ Negative life event score } \\
\hline Low (0-3) & $363(29.4)$ & $335(27.1)$ & $243(19.7)$ & I37 (II.I) & $158(12.8)$ & I,236 (45.6) \\
\hline Medium (4-9) & $96(7.6)$ & $212(16.8)$ & $239(18.9)$ & $295(23.4)$ & 421 (33.3) & I,263 (46.6) \\
\hline High (10-13) & $5(2.4)$ & $17(8.1)$ & $31(14.7)$ & $56(26.5)$ & $102(48.3)$ & $211(7.8)$ \\
\hline
\end{tabular}


Table 5 Migraine triggers

\begin{tabular}{ll}
\hline & Patients, $\mathbf{n}(\%)$ \\
\hline Identified triggers that may cause migraines & $2,036(78.2)$ \\
Stress & $\mathrm{I}, 557(76.7)$ \\
Environmental (eg, weather) & $\mathrm{I}, 589(78.3)$ \\
Lack of sleep & $\mathrm{I}, 382(68.1)$ \\
Hormones/menstrual cycle & $\mathrm{I}, \mathrm{I} 79(58 . \mathrm{I})$ \\
Missing meals & $\mathrm{I}, 096(54.0)$ \\
Certain smells & $\mathrm{I}, 058(52.1)$ \\
Alcohol/drugs & $709(34.9)$ \\
Physical activity & $603(29.7)$ \\
Sexual activity & $\mathrm{I} 65(8.1)$ \\
Other & $392(19.3)$ \\
Have taken steps to avoid triggers & $\mathrm{I}, 793(88.3)$ \\
\hline
\end{tabular}

$27.0 \%$ reported being "satisfied" with their current migraine treatment (see Figure 5). One-third of respondents (33.7\%) reported being satisfied with their treating physician and a further $29.6 \%$ were neutral on their satisfaction with their treating physician. Participant satisfaction with behavioral or complementary therapies was found to be predominantly neutral $(38.7 \%)$.

\section{Relationship between migraine measures and comorbid disorders}

The results of the Pearson's $R$ correlational analyses identifying relationships between the negative life events score, length of time since the onset of migraine symptoms, and the monthly frequency of migraine symptoms by presence of comorbid disorders can be found in Table 7. The negative life events score was found to be significantly correlated $(P<0.01$ or $P<0.05)$ with all comorbid diseases except for diabetes and chronic obstructive pulmonary disease. The reported length of time since first migraine symptoms was found to be significantly correlated $(P<0.01)$ for all disorders except stroke and epilepsy. Further, the reported frequency of migraine symptoms per month was found to be significantly correlated $(P<0.01$ or $P<0.05)$ for all disorders except for stroke. The negative life event score, the length of time since onset of migraine, and the monthly frequency of migraine symptoms were found to be significantly correlated with each other $(P<0.01)$. Additional analyses were conducted according to age and sex; however, these analyses were not found to be statistically significant. The negative live events score was separated into tertiles, ie, high (score 10-13), medium (score 4-9), and low (score 0-3) scores. Analyses of variance comparing these groups found that those in the higher tertile group of negative life impact were more likely to have a higher frequency of migraine symptoms, a longer history of migraine symptoms, and were more likely to experience comorbid disorders. The results of these analyses of variance can be found in Tables $8-10$.

\section{Discussion}

The present study characterized migraineurs and examined their treatment patterns, stress attributes, migraine disorder characteristics, and disability profile. As in previous research and clinical data, ${ }^{1}$ the vast majority of migraineurs in this study were female. Respondents in this survey tended to be younger than in other large migraine studies, with a higher proportion of participants aged 18-39 years but this is most likely due to the online mode of delivery rather than a change in the migraineur population..$^{13}$ Similar to previous findings, migraineurs at every degree of symptom frequency reported that their migraine symptoms negatively affected their lives. Migraineurs in this study were found to experience elevated levels of bodily pain, anxiety, and depression, and were able to identify physiological aspects of their migraines in proportions similar to those in other studies, ${ }^{14}$ but a substantial minority appeared to be ignorant of the prodromal features

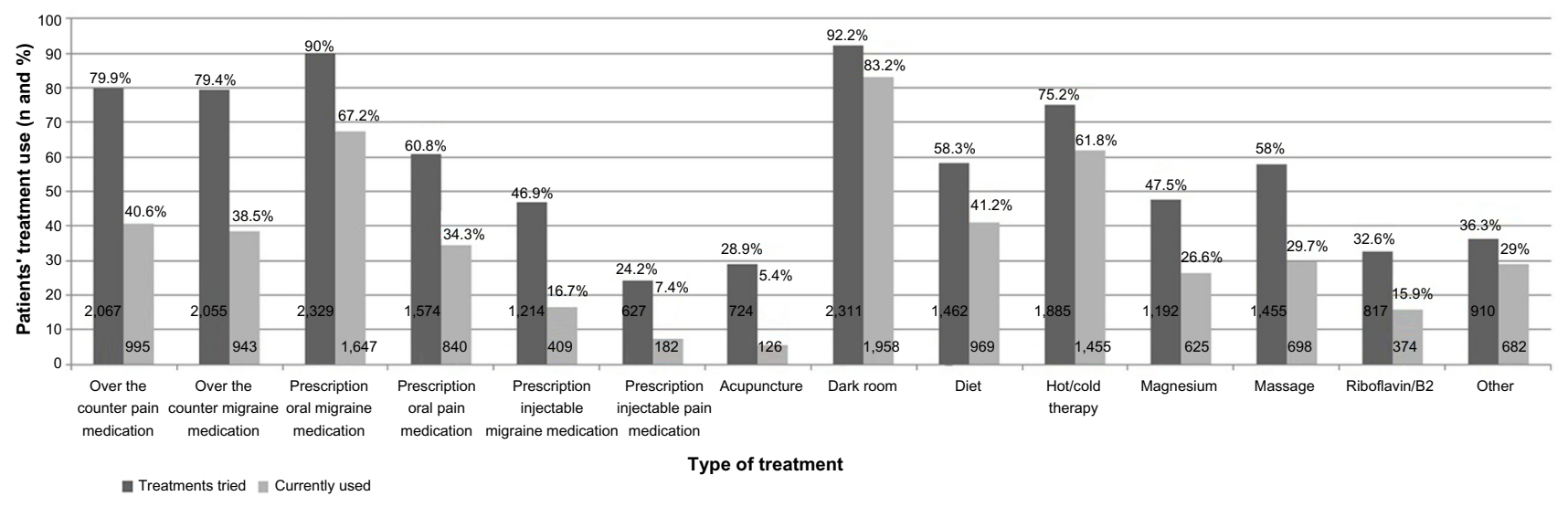

Figure 3 Rates of current and former treatment usage for migraine. 


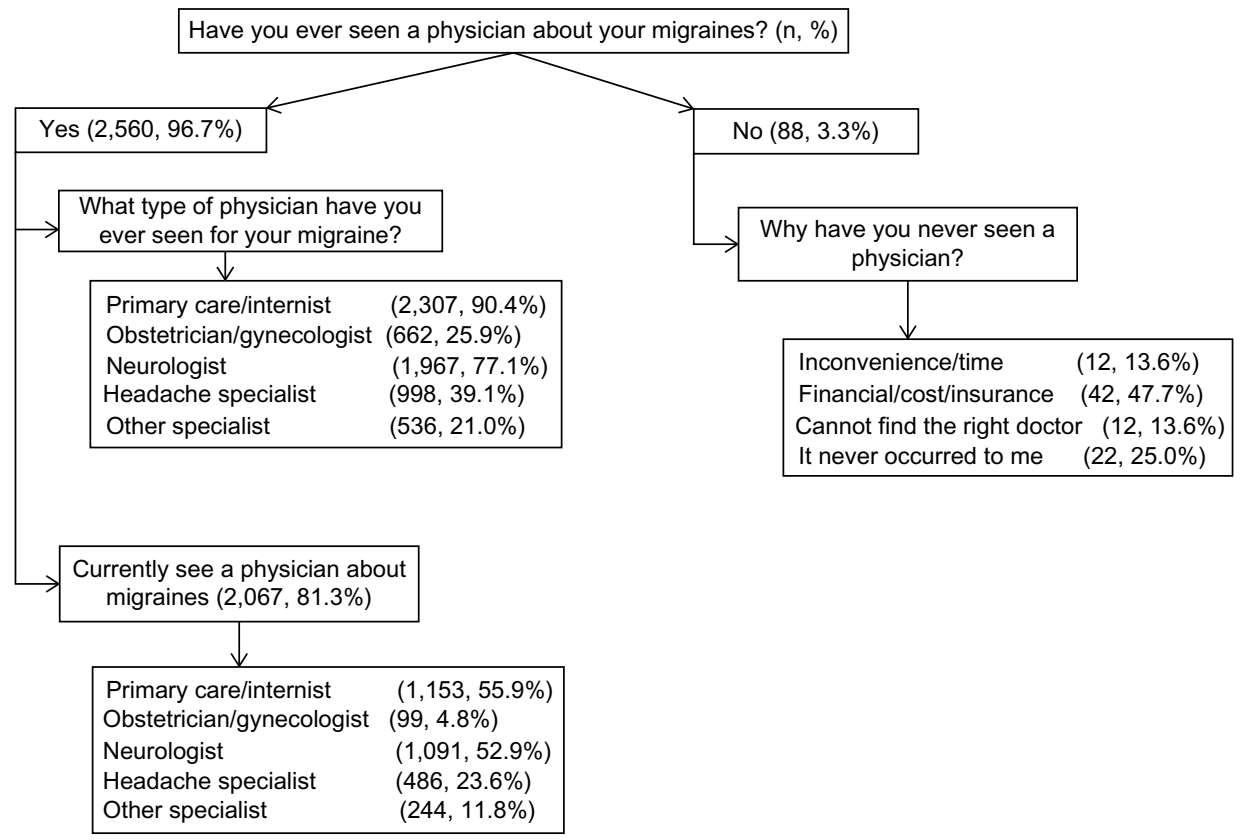

Figure 4 Migraine treatment practitioners.

of their condition. This is significant in that while migraine headaches commonly confer negative impacts, the precipitating factors are poorly understood by a large percentage of migraineurs, casting doubt on the ability of some patients to be knowledgeable about which treatment may be most efficacious for them. . $^{2,7,15}$

Similar to previous findings, migraineurs at every degree of symptom frequency reported that their migraine symptoms negatively affected their lives. The length of time since the participant's first migraine and the number of migraine symptoms were found to have a stronger relationship with negative interpersonal and situational impacts than with negative affective and psychological impacts, such as shame or embarrassment related to migraines. While this relationship may be a result of misperception of treatment by others or poor self-knowledge of emotional state, this observation is significant in that it suggests that migraineurs may attribute a wide variety of negative social interactions to their disorder rather than other factors, such as the dispositions of others

Table 6 Migraine treatment discontinuation

\begin{tabular}{ll}
\hline Reason for discontinuation of medicine use & Patient, n (\%) \\
\hline $\begin{array}{l}\text { Avoided using a medicine to treat migraines because } \\
\text { of side effects }\end{array}$ & $\mathrm{I}, 689(65.4)$ \\
$\begin{array}{l}\text { Stopped using a medicine to treat your migraines } \\
\text { because of a side effect }\end{array}$ & $\mathrm{I}, 709(66.2)$ \\
$\begin{array}{l}\text { Ever withheld taking medication to save for later } \\
\text { Ever avoided using a medication due to cost }\end{array}$ & $\mathrm{I}, 878(73.0)$ \\
\hline
\end{tabular}

or situational factors. Previous research has treated the stress resulting from migraines as a purely affective or psychological experience on the part of migraineurs; ${ }^{8}$ however, our research indicates that migraine-associated stress negatively impacts social and professional areas even more strongly than psychological states. While the findings of this study suggest that the negative impacts of migraine related to the number and frequency of migraine symptoms may be more closely related to the interpersonal impact of migraine, this may not be a simple static relationship. It is possible that the interpersonal impact of migraines may be more easily attributed to specific events whereas the self-perceived internal stress may result from a general sense derived from recent or distant personal experiences. Future research should further explore the relationship between the impact of migraines on an individual's social network and the negative affective and psychological impacts on migraineurs.

Despite the fact that the vast majority of the participants in this study sought the care of a physician for their migraine headaches, only a subset of these participants continued their treatment and an even smaller proportion were satisfied or extremely satisfied with their treatment. Our study found that migraineurs tended to be dissatisfied with their migraine treatment regardless of the intervention used. These results continue the trend of low patient satisfaction with migraine treatment found in previous studies; however, the current sample tended to be even more dissatisfied than noted in previous research. ${ }^{7,16}$ Similar to previous studies, the current study 


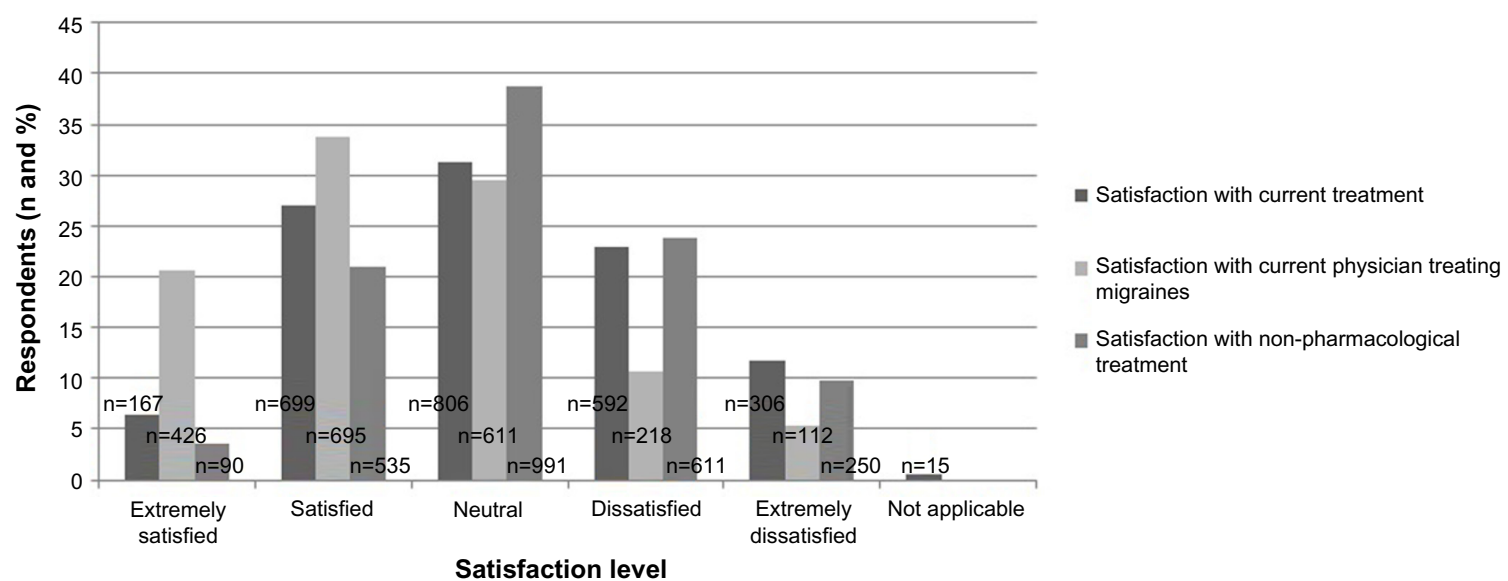

Figure 5 Migraineur satisfaction levels of medical treatment, treating physician, and non-pharmacological treatments.

found that migraineurs often attempt many treatments, but are discouraged by factors such as cost, side effects, and efficacy. ${ }^{17}$ Even for highly efficacious, cost-effective treatments, limited availability of treatment or lack of treatment providers may prevent an individual from learning about and using low-risk and potentially high-benefit practices. ${ }^{18}$ The finding that a proportion of migraineurs found some benefit for each treatment suggests that the differential effectiveness of intervention may be due to idiosyncratic patient characteristics rather than specific treatment ineffectiveness. This emphasizes the need for a migraine practitioner to understand the interaction between psychological, social, and biological characteristics in the individual patient in order to accurately match a patient with the treatment approach most suited to their specific disorder pattern and personal circumstances.

The most salient difference in how migraineurs perceived their disorder was in the symptomatic manifestation of the disease. Although migraine headaches are frequently associated with aura, our sample reported migraine with aura at a lower level than would have been anticipated. ${ }^{19}$ Respondents in this study reported symptoms of nausea/vomiting and sensitivity to light to a higher degree than has been found in previous studies. ${ }^{20}$ In addition, participants in our study reported migraine symptoms that have not been widely studied to date, such as puffy eyelids and food cravings. The variety of symptoms reported by migraineurs attests

Table 7 Pearson $R$ correlations between migraine characteristics and comorbid disorders

\begin{tabular}{llll}
\hline & $\begin{array}{l}\text { Negative life } \\
\text { event score }\end{array}$ & $\begin{array}{l}\text { Length of time since } \\
\text { first migraine symptoms }\end{array}$ & $\begin{array}{l}\text { Migraine symptoms } \\
\text { per month (n) }\end{array}$ \\
\hline Depression & $0.362^{* *}$ & $0.241^{* *}$ & $0.253^{* *}$ \\
Anxiety & $0.325^{* *}$ & $0.183^{* *}$ & $0.230^{* *}$ \\
Stroke & $0.037^{*}$ & 0.036 & 0.034 \\
Epilepsy & $0.040^{*}$ & 0.007 & $0.047^{*}$ \\
Hypertension & $0.067^{* *}$ & $0.113^{* *}$ & $0.101^{* *}$ \\
Diabetes & 0.034 & $0.054^{* *}$ & $0.052^{* *}$ \\
Bipolar & $0.077^{* *}$ & $0.055^{* *}$ & $0.042^{*}$ \\
IBS & $0.182^{* *}$ & $0.156^{* *}$ & $0.167^{* *}$ \\
Chronic pain & $0.346^{* *}$ & $0.186^{* *}$ & $0.326^{* *}$ \\
Fibromyalgia & $0.189^{* *}$ & $0.121^{* *}$ & $0.171^{* *}$ \\
Thyroid disease & $0.117^{* *}$ & $0.110^{* *}$ & $0.123^{* *}$ \\
Chronic fatigue & $0.267^{* *}$ & $0.146^{* *}$ & $0.253^{* *}$ \\
Asthma & $0.132^{* *}$ & $0.096^{* *}$ & $0.135^{* *}$ \\
COPD & -0.002 & $0.049^{* *}$ & $0.050^{* *}$ \\
Autoimmune disorder & $0.087^{* *}$ & $0.062^{* *}$ & $0.067^{* *}$ \\
Negative life event score & 1 & $0.299^{* *}$ & $0.519^{* *}$ \\
Length of time since first migraine symptoms & $0.299^{* *}$ & 1 & $0.406^{* *}$ \\
Migraine symptoms per month (n) & $0.519^{* *}$ & $0.406^{* *}$ & 1 \\
\hline
\end{tabular}

Notes: ${ }^{* *}$ Correlation is significant at the 0.01 level (two-tailed); *correlation is significant at the 0.05 level (two-tailed).

Abbreviations: COPD, chronic obstructive pulmonary disease; IBS, irritable bowel syndrome. 
Table 8 Analysis of variance of negative life events score triad by comorbid disorders

\begin{tabular}{llll}
\hline & F (df-btw, df-tot) & P-value & $\eta^{2}$ \\
\hline Depression & $359.397(\mathrm{I}, 2,909)$ & 0.000 & 0.110 \\
Anxiety & $261.978(\mathrm{I}, 2,909)$ & 0.000 & 0.083 \\
Stroke & $2.432(\mathrm{I}, 2,909)$ & 0.119 & $0.00 \mathrm{I}$ \\
Epilepsy & $4.005(\mathrm{I}, 2,909)$ & 0.045 & $0.00 \mathrm{I}$ \\
Hypertension & $8.876(\mathrm{I}, 2,909)$ & 0.003 & 0.003 \\
Diabetes & $5.675(\mathrm{I}, 2,909)$ & 0.017 & 0.002 \\
Bipolar & $15.787(\mathrm{I}, 2,909)$ & 0.000 & 0.005 \\
IBS & $82.398(\mathrm{I}, 2,909)$ & 0.000 & 0.028 \\
Chronic pain & $308.965(\mathrm{I}, 2,909)$ & 0.000 & 0.096 \\
Fibromyalgia & $79.536(\mathrm{I}, 2,909)$ & 0.000 & 0.027 \\
Thyroid disease & $30.062(\mathrm{I}, 2,909)$ & 0.000 & 0.010 \\
Chronic fatigue & $\mathrm{I} 89.367(\mathrm{I}, 2,909)$ & 0.000 & $0.06 \mathrm{I}$ \\
Asthma & $37.65 \mathrm{I}(\mathrm{I}, 2,909)$ & 0.000 & 0.013 \\
COPD & $0.109(\mathrm{I}, 2,909)$ & $0.74 \mathrm{I}$ & 0.000 \\
Autoimmune disorder & $\mathrm{I} 6.68 \mathrm{I}(\mathrm{I}, 2,909)$ & 0.000 & 0.006 \\
Length of time since first & $53.626(4,2,909)$ & 0.000 & 0.069 \\
migraine symptoms & & & \\
Migraine symptoms per month (n) & $\mathrm{I} 60.685(5,2,909)$ & 0.000 & 0.217 \\
\hline
\end{tabular}

Abbreviations: COPD, chronic obstructive pulmonary disease; IBS, irritable bowel syndrome.

to the individualized nature of the disorder. Further, the diverse manifestations of migraine symptoms lends further support to the view that migraines must be considered in the context of the individual, because different symptoms may have varying degrees of impact on an individual's daily life. It is not unreasonable to consider symptoms such as vomiting and sensitivity to light as potentially eliciting a greater change in a sufferer's behavior than symptoms such as food cravings. Different symptoms may exert differential

Table 9 Analysis of variance for length of time since first migraine symptom by comorbid disorders

\begin{tabular}{llll}
\hline & F (df-btw, df-tot) & P-value & $\eta^{2}$ \\
\hline Depression & $192.034(I, 2,909)$ & 0.000 & 0.062 \\
Anxiety & $120.116(I, 2,909)$ & 0.000 & 0.040 \\
Stroke & $4.015(I, 2,909)$ & 0.045 & $0.00 I$ \\
Epilepsy & $0.607(I, 2,909)$ & 0.436 & 0.000 \\
Hypertension & $38.634(I, 2,909)$ & 0.000 & 0.013 \\
Diabetes & $7.148(I, 2,909)$ & 0.008 & 0.002 \\
Bipolar & $8.989(I, 2,909)$ & 0.003 & 0.003 \\
IBS & $73.210(I, 2,909)$ & 0.000 & 0.025 \\
Chronic pain & $107.060(I, 2,909)$ & 0.000 & 0.036 \\
Fibromyalgia & $39.812(I, 2,909)$ & 0.000 & 0.014 \\
Thyroid disease & $35.87 I(I, 2,909)$ & 0.000 & 0.012 \\
Chronic fatigue & $64.419(I, 2,909)$ & 0.000 & 0.022 \\
Asthma & $30.355(I, 2,909)$ & 0.000 & 0.010 \\
COPD & $6.326(I, 2,909)$ & 0.012 & 0.002 \\
Autoimmune disorder & $12.335(I, 2,909)$ & 0.000 & 0.004 \\
Negative life event score H, M, L & $107.144(2,2,909)$ & 0.000 & 0.069 \\
Migraine symptoms per month $(\mathrm{n})$ & $1028.578(5,2,909)$ & 0.000 & 0.639 \\
\hline Abbrat & &
\end{tabular}

Abbreviations: COPD, chronic obstructive pulmonary disease; IBS, irritable bowel syndrome; $\mathrm{H}$, high; M, Medium; L, low.
Table 10 Analysis of variance for migraine frequency by comorbid disorders

\begin{tabular}{llll}
\hline & F (df-btw, df-tot) & P-value & $\eta^{2}$ \\
\hline Depression & $198.174(I, 2,909)$ & 0.000 & 0.064 \\
Anxiety & $162.657(I, 2,909)$ & 0.000 & 0.053 \\
Stroke & $3.373(I, 2,909)$ & 0.066 & $0.00 I$ \\
Epilepsy & $6.553(I, 2,909)$ & $0.0 I I$ & 0.002 \\
Hypertension & $29.710(I, 2,909)$ & 0.000 & 0.010 \\
Diabetes & $8.025(I, 2,909)$ & 0.005 & 0.003 \\
Bipolar & $5.258(I, 2,909)$ & 0.022 & 0.002 \\
IBS & $83.857(I, 2,909)$ & 0.000 & 0.028 \\
Chronic pain & $345.932(I, 2,909)$ & 0.000 & 0.106 \\
Fibromyalgia & $87.74 I(I, 2,909)$ & 0.000 & 0.029 \\
Thyroid disease & $44.674(I, 2,909)$ & 0.000 & 0.015 \\
Chronic fatigue & $199.614(I, 2,909)$ & 0.000 & 0.064 \\
Asthma & $53.744(I, 2,909)$ & 0.000 & 0.018 \\
COPD & $7.160(I, 2,909)$ & 0.007 & 0.002 \\
Autoimmune disorder & $I 2.975(I, 2,909)$ & 0.000 & 0.004 \\
Negative life event score H, M, L & $424.84 I(2,2,909)$ & 0.000 & 0.226 \\
Length of time since first & $24 I .488(4,2,909)$ & 0.000 & 0.250 \\
migraine symptoms & & & \\
\hline Abbreviatons: COPD, chronc & & & \\
\hline
\end{tabular}

Abbreviations: COPD, chronic obstructive pulmonary disease; IBS, irritable bowel syndrome; $\mathrm{H}$, high; M, medium; L, low.

degrees of impact on a sufferer's day-to-day life, even when experienced at similar frequencies, and as mentioned previously, the individual characteristics of the migraineur may determine the level of stress experienced as a result of these symptoms.

The present study probed the areas of daily life impacted by migraine headaches and found that these headaches impose a substantial biological, psychological, and social burden on migraineurs. The pervasive and destructive impact of migraine headaches on a migraineurs' life has been well documented in the literature, but previous research has not closely examined how the stress of these negative impacts is moderated by the time since onset and the frequency of the disorder. ${ }^{7}$ The stress of chronic migraine may be a cyclical process which contributes to the development and fermentation of comorbid disorders, additional stress, and more migraines. This insidious cycle may be exacerbated by a higher number of symptoms, a higher migraine frequency, or pre-existing psychological or medical conditions. The psychological impact of migraines may inhibit the ability to engage in healthy practices that would reduce the prevalence of other disorders, decrease the use of adaptive coping with biological symptoms, exacerbate the pain experience, and may discourage adherence to treatment. Psychological distress, as well as pain, have been found to be strongly associated with development of depression or anxiety disorders, ${ }^{21}$ and these were found to be the most common comorbid disorders in our study. 
The high medical and personal burden reported by participants in the present study is significant in terms of emphasizing the pervasive onus that migraine headaches confer. This raises the question of how much medical misery can someone endure? Competing health priorities created by comorbid diseases can exacerbate the everyday cost of migraine symptoms by inhibiting a migraineur's ability to cope biologically and psychologically. High levels of stress have been shown to lead to a higher prevalence of an array of medical and psychological disorders. ${ }^{22}$ This is true of both depression and anxiety, which have both been found to be more prevalent in the migraineur population..$^{23}$ The findings of this study underscore the clinical importance of understanding the social and psychological impact of migraine headaches on a migraineur. Participants found to have a higher degree of stress attributable to migraine symptoms were also found to have more comorbid disorders, and importantly, the most common comorbid disorders found in this sample are also known to be highly susceptible to stress.

Our results highlight the clinical relevance of understanding the impacts of migraine headaches on patients. Previous research has identified chronic psychological stress, such as in sufferers of a chronic disorder, to be a major suppressor of the human immune system. ${ }^{24}$ Healthy social relationships may serve as a protective factor with regard to the development of additional disorders in an already medically and psychologically vulnerable population; however, our study sample reported that they experienced a variety of negative impacts to their social relationships due to migraine, which suggests that migraineurs may lack social capital when compared with other chronically ill groups. Use of adaptive coping strategies and healthy lifestyle choices can also act as protective factors for individuals with migraine headaches. The active engagement with biological, psychological, and social protective factors can help to reduce the "suffering" experienced by migraineurs and give patients more control over their headache pain and symptoms. Migraine practitioners should make efforts to understand and alleviate the impact of stress on the diverse areas of life in migraineurs. Where this is beyond the scope of the prescriber's medical practice, they should consider referrals to pain psychology specialists, other mental health providers with specific training in the biopsychosocial models of pain treatment, or pain support groups.

\section{Limitations}

There are some limitations to the present study. The principle limitation is the bias, such as self-selection and selfidentification, inherent in studying an online sample. The lack of information collected relating to participant race, education, and socioeconomic status also limit the generalizability of this study.

\section{Conclusion}

The present study extends the body of literature characterizing treatment patterns, disorder characteristics, and disability profile in migraineurs. Migraine-associated stress was found to be a much more diverse experience than previous researchers have described. Negative social and personal impacts of migraines were found to be common in this sample. Migraineurs were found to have tried many different treatments but were, overall, unsatisfied with their efficacy. The highly personal experience of migraine is seen in the variety of negative life impacts secondary to migraine headache, diverse comorbid disorder profiles, and variable responses to migraine treatments. Each of these factors contributes to the idiosyncratic experience of migraine. Practitioners must understand the personal experience of each migraineur in order to match their patient to a treatment which will alleviate the social, biological, and psychological experiences of migraine headache. Migraine headaches were found to impart a wide variety of possible negative impacts on an individual's life. Migraineurs who experienced more frequent symptoms tended to experience more negative life events related to migraine, as well as more medical or psychiatric comorbidities. The vast majority of participants reported having seen a physician about their migraine headaches, with financial concerns being the most commonly reported reason as to why participants had not contacted a physician about their migraines. Similarly, many participants had attempted various medications in the past; however, these medications were frequently discontinued due to side effects, and the cost of medication was reported to be the most common reason for discontinuation. While our study sample reported common migraine symptoms in similar proportions to previous research, some symptoms reported by our study participants, such as puffy eyelids and food cravings, have not been examined in previous migraine research. Future research into migraine headaches should explore the social network impacts of migraine headache and examine this impact on the effectiveness of treatment and delivery of care for migraineurs.

\section{Acknowledgment}

Partial funding for this research was provided by the National Institutes of Health (K23DA03039, awarded to AW). The 
content is solely the responsibility of the authors and does not necessarily represent the official views of the National Institutes of Health.

\section{Disclosure}

$\mathrm{CDM}, \mathrm{ABW}$, and $\mathrm{AB}$ declare that they have no competing interests to report. AB is an employee of Health Union, LLC, which owns Migraine.com. No other relationship, bias, or ethical conflict exists among the authors of this work.

\section{References}

1. Smitherman TA, Burch R, Sheikh H, Loder E. The prevalence, impact, and treatment of migraine and severe headaches in the United States: a review of statistics from national surveillance studies. Headache. 2013;53:427-436.

2. Bigal ME, Lipton RB. The epidemiology, burden, and comorbidities of migraine. Neurol Clin. 2009;27:321-334.

3. DeMaagd G. The pharmacological management of migraine, part 1. P T. 2008;33:404-416.

4. Gaul C, Christmann N, Shroder D, et al. Differences in clinical characteristics and frequency of accompanying migraine features in episodic and chronic cluster headache. Cephalalgia. 2012;32:571-577.

5. Pesaturo KA, Wooding FG. Modern management of the migraine headache. Am J Lifestyle Med. 2009;3:147-159.

6. Penzien DB, Rains JC, Andrasik F. Behavioral management of recurrent headache: three decades of experience and empiricism. Appl Psychophysiol Biofeedback. 2002;27:163-181.

7. Bigal ME, Serrano D, Reed M, Lipton RB. Chronic migraine in the population: burden, diagnosis, and satisfaction with treatment. Neurology. 2008;71:559-566.

8. Wacogne C, Lacoste JP, Guillibert E, Hugues FC, Le Jeunne C. Stress, anxiety, depression, and migraine. Cephalalgia. 2003;23:451-455.

9. Holm J, Lokken C, Myers T. Migraine and stress: a daily examination of temporal relationships in women migraineurs. Headache. 1997;37: 553-558.
10. Sauro $\mathrm{K}$, Becker $\mathrm{W}$. The stress and migraine interaction. Headache. 2009;49:1378-1386.

11. Strine TW, Chapman DP, Balluz LS. Population-based US study of severe headaches in adults: psychological distress and comorbidities. Headache. 2006;46:223-232.

12. Migraine.com [homepage on the internet]. Health Union, LLC. Available at: http://migraine.com/. Accessed April 24, 2014.

13. Bigal ME, Borucho S, Serrano D, Lipton RB. The acute treatment of episodic and chronic migraine in the USA. Cephalalgia. 2008;29: 891-897.

14. Wachholtz A, Pargament K. Migraines and meditation: does spirituality matter? J Behav Med. 2008;31:351-366.

15. Lamber GL, Zagami AS. The mode of action of migraine triggers: a hypothesis. Headache. 2008;49:253-275.

16. Demarin V, Vukovic V, Ivankovic M. Scientific approach to migraine Med Sci. 2009;33:43-52.

17. Elrington G. Migraine: diagnosis and management. J Neurol Neurosurg Psychiatry. 2002;72 Suppl 2:ii10-ii15.

18. Tonelli M, Wachholtz A. Meditation based treatment yielding immediate relief for meditation naïve migraineurs. Pain Manag Nurs. 2014;15:36-40.

19. Kunkel RS. Migraine aura without headache: benign, but a diagnosis of exclusion. Cleve Clin J Med. 2002;72:529-534.

20. Moens G, Johannik K, Verbeek C, Bulterys S. The prevalence and characteristics of migraine among the Belgian working population. Acta Neurol Belg. 2007;107:84-90.

21. Korff MV, Gregory S. The relationship between pain and depression. Br J Psychiatry. 1996;168:101-108.

22. Boscarino JA. Diseases among men 20 years after exposure to severe stress: Implications for clinical research and medical care. Psychosom Med. 1997;59:605-614.

23. Breslau N, Davis GC, Andreski P. Migraine, psychiatric disorders, and suicide attempts: an epidemiologic study of young adults. Psychiatry Res. 1991;37:11-23.

24. Segerstrom SC, Miller GE. Psychological stress and the human immune system: a meta-analytic study of 30 years of inquiry. Psychol Bull. 2004;130:601-630.
Journal of Pain Research

\section{Publish your work in this journal}

The Journal of Pain Research is an international, peer-reviewed, open access, online journal that welcomes laboratory and clinical findings in the fields of pain research and the prevention and management of pain. Original research, reviews, symposium reports, hypothesis formation and commentaries are all considered for publication.

\section{Dovepress}

The manuscript management system is completely online and includes a very quick and fair peer-review system, which is all easy to use. Visit http://www.dovepress.com/testimonials.php to read real quotes from published authors. 